\title{
Calendar of Short Courses \& Workshops
}

\author{
Gang Wang \\ Research and Development Center for Functional Crystals, Institute of Physics, Chinese Academy of Sciences, No. 8 Nansanjie, Zhongguancun, \\ Haidian District Beijing 100190, China \\ gangwang@iphy.ac.cn
}

25 March-2 April 2017

Sixteenth BCA/CCG Intensive Teaching School in X-Ray Structure Analysis Trevelyan College, Durham, UK [Info: http://community.dur.ac.uk/durham.X-ray-school/staff. $\mathrm{htm}]$

\section{7-9 April 2017}

Fourth School on Crystal Structure Determination from Diffraction Data: Application on Powder Samples Hammamet, Tunisia [Info: http://www.iucr.org/calendar/ events/types/schools/4th-school-on-crystal-structuredetermination-from-diffraction-data-application-on-powdersamples]

\section{4-28 April 2017}

Practical X-ray Fluorescence ICDD Headquarters, Newtown Square, Pennsylvania, USA [Info: http://www. icdd.com/education/xrf.htm]

8-19 May 2017

Macromolecular Crystallography School 2017: Structural Biology to Enhance High-Impact Research in Health and Disease Montevideo, Uruguay [Info: http://www.iucr.org/calendar/events/types/schools/macromolecular-crystallographyschool-2017-structural-biology-to-enhance-high-impact-researchin-health-and-disease]

\section{0-24 May 2017}

Modern Trends in Mathematical Crystallography Second Manila International Workshop on Mathematical Crystallography Manila, Philippines [Info: http://www.crystallography.fr/mathcryst/manila2017.php]

29 May-2 June 2017

To.Sca.Lake 2.0: Total Scattering for Nanotechnology on the Como Lake (co-sponsored by ICDD) Como, Italy [Info: http://tsnl.lakecomoschool.org/]

29 May-2 June 2017

Sixth International School on Biological Crystallization Granada, Spain [Info: http://www.isbcgranada.org/]

2-11 June 2017

International School of Crystallography (CrystalErice 50th: Integrative Structural Biology) Erice, Italy [Info: https://crystalerice.org/2017/]

5-9 June 2017

Fundamentals of X-ray Powder Diffraction ICDD
Headquarters, Newtown Square, Pennsylvania, USA [Info: http://www.icdd.com/education/xrd.htm]

\section{5-7 June 2017}

canSAS: Small Angle Scattering Workshop San Francisco, California, USA [Info:https://sites.google.com/a/lbl.gov/cansas/home]

11-17 June 2017

Shanghai International Crystallographic School working with Bilbao Crystallographic Server Shanghai, China [Info: http://icqms.shu.edu.cn/abc2017/]

\section{1-22 June 2017}

Zurich School of Crystallography 2017 University of Zurich, Zurich, Switzerland [Info: http://www.chem.uzh.ch/ linden/zsc/]

\section{2-16 June 2017}

Advanced Methods in X-ray Powder Diffraction ICDD Headquarters, Newtown Square, Pennsylvania, USA [Info: http://www.icdd.com/education/xrd.htm]

2-7 July 2017

Fourth European Crystallographic School (ECS4) Warsaw, Poland [Info: http://ecs4.ecanews.org/]

\section{4-15 August 2017}

International Workshop on Improving Data Quality in XAFS Spectroscopy (Q2XAFS2017) Diamond Light Source, Oxfordshire, UK [Info: http://www.diamond.ac.uk/ Home/Events/2017/Q2XAFS2017.html]

\section{5-20 August 2017}

Crystallographic Computing School Bangalore, India [nfo: http://www.iucr.org/resources/commissions/crystallographiccomputing/schools/bangalore2017]

30 August-4 September 2017

International School on Fundamental Crystallography and Workshop on Structural Phase Transitions Odisha, India [Info: http://www.iucr2017.org/wp-content/uploads/ 2016/09/School.pdf]

\section{5-29 September 2017}

Basic \& Advanced Rietveld Refinement \& Indexing Workshop ICDD Headquarters, Newtown Square, Pennsylvania, USA [Info: http://www.icdd.com/education/ rietveld-workshop.htm] 